\title{
Congenital malformations and perinatal complications in infants of patients with bipolar and schizoaffective disorders treated with anticonvulsants: a systematic review
}

A. Giménez Palomo ${ }^{1}$, I. Pacchiarotti ${ }^{1}$, J. Gili ${ }^{1}$, A. Murru ${ }^{1}$, S. Gomes ${ }^{1}$, J. Pinzón ${ }^{1}$,

G. Anmella ${ }^{1}$, M. Gómez-Ramiro' ${ }^{1}$, N. Verdolini ${ }^{1}$, M. Valentí ${ }^{1}$, J.M. Goikolea ${ }^{1}$, E. Vieta ${ }^{1}$

${ }^{1}$ Hospital Clínic de Barcelona

agimenezp@clinic.cat

\section{INTRODUCTION}

Antiepileptic drugs (AEDs) are commonly used as a treatment for acute phases and prevention of relapses in bipolar disorder (BD) and schizoaffective disorder (SAD). They have been often associated to adverse outcomes in pregnancy and major congenital malformations (MCM), but most studies have been focused on patients with epilepsy and little data is available about psychiatric patients.

The aim of this study was to summarize through a systematic search the available evidence about the adverse effects of AEDs in infants when they are used in pregnant patients with BD or SAD.

\section{METHODS}

We searched four databases from inception to 18-January-2019. We included peer-reviewed observational studies on the use of AEDs in pregnant women with BD or SAD. We excluded studies not reporting data on BD or SAD, not specifying the AED or not assessing pregnancy outcomes or major congenital malformations.

Databases: PubMed $\rightarrow$ Cochrane $\rightarrow$ PsycINFO/PsycARTICLES $\rightarrow$ ClinicalTrials.gov

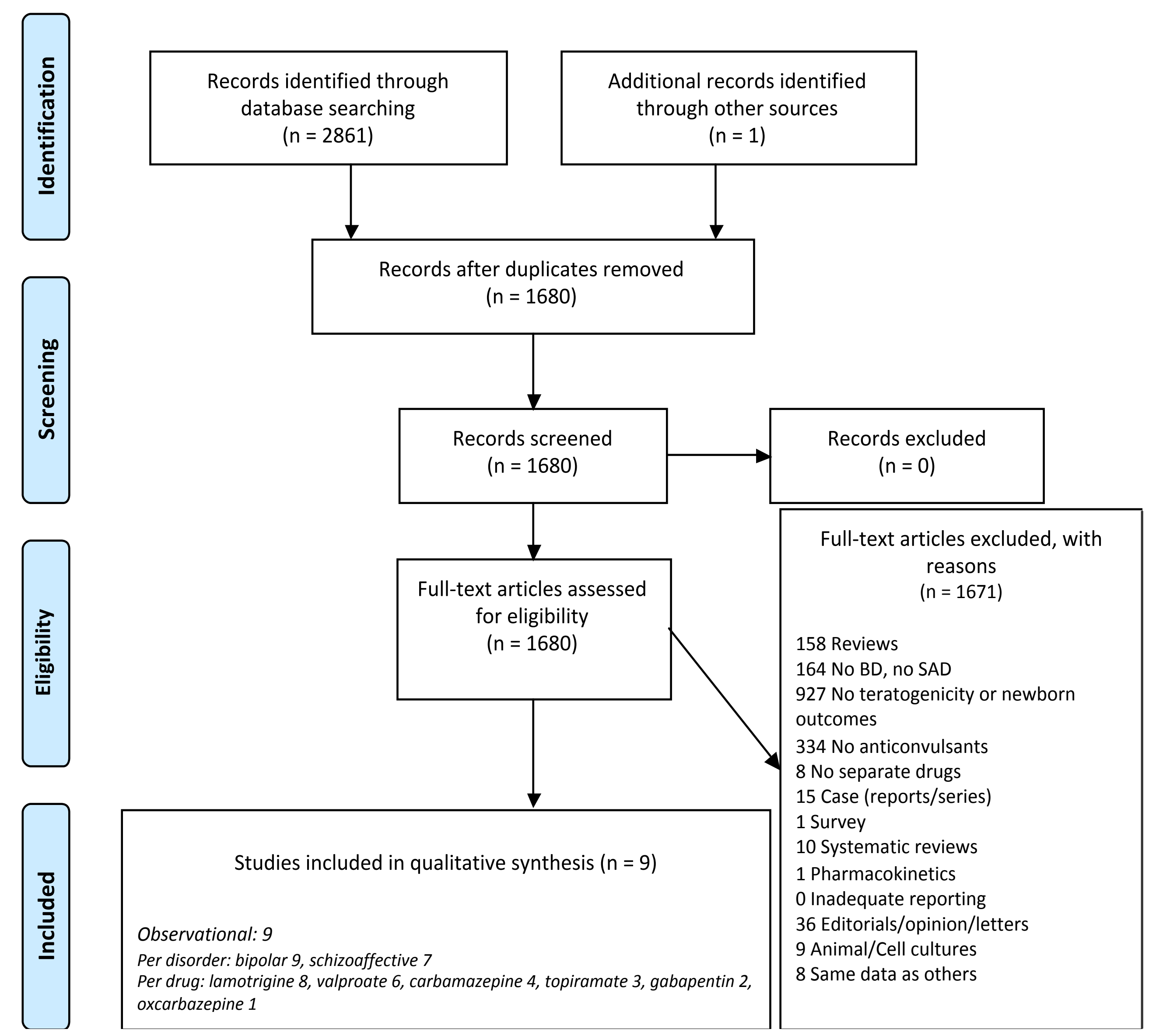

\section{RESULTS}

The pooled records amounted to 2,861. After duplicate removal and inclusion/exclusion criteria application, we included 9 observational studies assessing patients with BD and SAD. The AEDs evaluated were lamotrigine, valproate, carbamazepine, oxcarbazepine, topiramate and gabapentin.

We found that lamotrigine is the drug with more data in patients with bipolar disorder. Valproate and carbamazepine were the AEDs most commonly associated to MCM. Overall, it maybe underlined that, at least with valproate use, the risk of MCM appears to be dose dependent and this would also explain the higher rate of teratogenic effects in epileptic women in which notoriously higher doses are used than in women with affective disorders. Lamotrigine showed the best safety profile. Higher rates of complications during pregnancy were observed in treated and untreated women with $\mathrm{BD}$ compared to healthy controls.

With respect to complications during pregnancy or the perinatal period, the included studies showed increased risks of caesarean delivery, instrumental delivery, a non-spontaneous start to delivery, and preterm delivery in women with $B D$ receiving or not AED treatment with valproate, lamotrigine or carbamazepine, with no differences between these drugs. Similarly, other studies showed a higher rate of spontaneous abortions in patients treated with AEDs.

\section{CONCLUSIONS}

AEDs may produce adverse outcomes in pregnancy and MCM in children of pregnant women with BD or SAD, showing higher risks at higher doses. Lamotrigine could be considered in this type of patients, given the low rate of adverse events. Valproate and carbamazepine use should be avoided during pregnancy.

The level of evidence for the use of AEDs in the maintenance treatment of BD and SAD during pregnancy is still limited, considering the lack of randomized controlled clinical trials mainly due to ethical aspects.

In addition, comparative studies between treated and untreated women with $\mathrm{BD}$ could strengthen the evidence on the effects of AEDs use in this specific population. Further research should also assess the effect of specific AEDs other than valproate and carbamazepine, such as lamotrigine, to investigate their safety profile, which could increase the accuracy of current recommendations. 